Proof. Let the circles $A E C$ and $A B D$ cut $A O$ in $X$ and $Y$ respectively. Then each of the angles $X E C, X C E, Y D B, Y B D$ is in the same segment of a circle as $O A B$ or $O A C$, and is equal to $\frac{1}{2} A$. Consequently the triangles $X E C, Y D B$ are congruent, and the four lines $X E, X C, Y D, Y B$ are equal. It also follows that $X E$ is a tangent to the circle $E A O$, and $Y D$ to $D A O$, so that, $X O . X A$ $=X E^{2}=Y D^{2}=Y O . Y A$. Accordingly $X$ and $Y$ must coincide, at the centre of a circle $B C D E$. In this circle the equal chords $B D$ and $C E$ subtend equal angles at the circumference. Therefore angles $B$ and $C$ are equal (or supplementary: this is impossible), and the triangle is isosceles.

74 Serpentink Avende, Ballsbridge, Dublin.

\title{
A note on equilateral polygons
}

\author{
By A. D. Russell.
}

Theorem. If a circle cut all the sides (produced if necessary) of an equilateral polygon, the algebraic sum of the intercepts between the vertices and the circle is zero; i.e., if any side $A B$ of the polygon be cut by the circle in $P$ and $Q$, then $\Sigma(A P+B Q)=0$, the intercepts being signed by fixing a positive direction round the contour of the polygon.

This was proved in a previous note, ${ }^{1}$ using Euclid III. 35, for the special case of a regular polygon; but the same proof applies to any equilateral polygon, which may be re-entrant or self-crossing.

Corollary. If $n$ chords $P_{i} O Q_{i}(i=1, \ldots, n)$ of a circle are drawn through a point $O$ parallel to the sides of an equilateral $n$-gon, the

1 "Theorem regarding a regular polygon and a circle cutting its sides," Mathematical Notes, No, 22, 1924. 
A. D. Russell

algebraic sum of their $2 n$ segments, $\Sigma\left(O \dot{P}_{i}+O Q_{i}\right)$, is zero. (As before, the segments are signed according to a positive direction round the contour of the polygon.)

This is a limiting case of the theorem, deduced by considering a variable polygon whose sides tend to zero while remaining parallel to those of the given polygon.

Trigonometrical Interpretation. Let the exterior angles of the equilateral $n$-gon (i.e:, the angles between the positive directions of consecutive sides) be $a_{1}, a_{2}, \ldots, a_{n}$. These are also the angles between the consecutive chords $P_{1} O Q_{1}, P_{2} O Q_{2}, \ldots, P_{n} O Q_{n}$. If the first of these chords makes $\theta$ with $O C$, where $C$ is the centre, then, paying due attention to sign,

$O C \cos \theta=\frac{1}{2}\left(O P_{1}+O Q_{1}\right), \quad O C \cos \left(\theta+a_{1}\right)=\frac{1}{2}\left(O P_{2}+O Q_{2}\right), \quad$ etc. ; and the Corollary gives $\cos \theta+\cos \left(\theta+\alpha_{1}\right)+\cos \left(\theta+\alpha_{1}+\alpha_{2}\right)+\ldots+\cos \left(\theta+\alpha_{1}+\alpha_{2}+\ldots+\alpha_{n-1}\right)=0$.

Since $\theta$ depends on the position of $O$, which is arbitrary, this is true for all values of $\theta$. Replacing $\theta$ by $\theta-\frac{1}{2} \pi$, it follows that

$\sin \theta+\sin \left(\theta+a_{1}\right)+\sin \left(\theta+a_{1}+\alpha_{2}\right)+\ldots+\sin \left(\theta+a_{1}+a_{2}+\ldots+a_{n-1}\right)=0$.

Formulae involving the interior angles $A_{1}=\pi-a_{1}$, etc., may be deduced. As a verification, taking $\theta=0$ in the case of a rhombus $(n=4)$, we have the obviously correct results

$$
\begin{array}{r}
1-\cos A_{1}+\cos \left(A_{1}+A_{2}\right)-\cos \left(A_{1}+A_{2}+A_{3}\right)=0, \\
\sin A_{1}-\sin \left(A_{1}+A_{2}\right)+\sin \left(A_{1}+A_{2}+A_{3}\right)=0 .
\end{array}
$$

12 HeUgh Street, FALKIRK. 